\title{
Prevalence of Sugar-Sweetened Beverage Intake Among Adults - 23 States and the District of Columbia, 2013
}

\author{
Sohyun Park, $\mathrm{PhD}^{1}$; Fang Xu, $\mathrm{PhD}^{2}$; Machell Town, $\mathrm{PhD}^{2}$; Heidi M. Blanck, $\mathrm{PhD}^{1}$
}

The 2015-2020 Dietary Guidelines for Americans recommend that the daily intake of calories from added sugars not exceed $10 \%$ of total calories.* Sugar-sweetened beverages (SSBs) are significant sources of added sugars in the diet of U.S. adults and account for approximately one third of added sugar consumption (1). Among adults, frequent (i.e., at least once a day) SSB intake is associated with adverse health consequences, including obesity, type 2 diabetes, and cardiovascular disease (2). According to the 2009-2010 National Health and Nutrition Examination Survey (NHANES), an in-person and phone follow-up survey, $50.6 \%$ of U.S. adults consumed at least one SSB on a given day (3). In addition, SSB intake varies by geographical regions $(4,5)$ : the prevalence of daily SSB intake was higher among U.S. adults living in the Northeast (68.4\%) and South $(66.7 \%)$ than among persons living in the Midwest (58.8\%). In 2013, the Behavioral Risk Factor Surveillance System (BRFSS), a telephone survey, revised the SSB two-item optional module to retain the first question on regular soda and expand the second question to include more types of SSBs than just fruit drinks. Using 2013 BRFSS data, self-reported SSB (i.e., regular soda, fruit drinks, sweet tea, and sports or energy drinks) intake among adults (aged $\geq 18$ years) was assessed in 23 states and the District of Columbia (DC). The overall age-adjusted prevalence of SSB intake $\geq 1$ time per day was $30.1 \%$ and ranged from $18.0 \%$ in Vermont to $47.5 \%$ in Mississippi. Overall, at least once daily SSB intake was most prevalent among adults aged $18-24$ years $(43.3 \%)$, men (34.1\%), non-Hispanic blacks (blacks) (39.9\%), unemployed adults (34.4\%), and persons with less than a high school education (42.4\%). States can use the data for program evaluation and monitoring trends, and information on disparities in SSB consumption could be used to create targeted intervention efforts to reduce SSB consumption.

\footnotetext{
*http://health.gov/dietaryguidelines/2015
}

BRFSS is a state-based, random-digit-dialed telephone survey of U.S. adults aged $\geq 18$ years, conducted annually by $\mathrm{CDC}$ and state health departments to monitor health conditions and behaviors related to public health concerns. ${ }^{\dagger}$ BRFSS uses multistage, stratified sampling to select a representative sample of the noninstitutionalized adult population in each state, DC, and three U.S. territories (American Samoa, the Federated States of Micronesia, and Guam). In 2013, the median response rate across all states was $46.4 \%$ from

${ }^{\dagger}$ http://www.cdc.gov/brfss/annual_data/annual_2013.html.

\section{INSIDE}

175 Outbreak of Foodborne Botulism Associated with Improperly Jarred Pesto — Ohio and California, 2014

178 Evaluation of Routine HIV Opt-Out Screening and Continuum of Care Services Following Entry into Eight Prison Reception Centers - California, 2012

182 Update: Interim Guidelines for Health Care Providers Caring for Infants and Children with Possible Zika Virus Infection — United States, February 2016

188 Notes from the Field: Ebola Virus Disease Response Activities During a Mass Displacemnt Event After Flooding - Freetown, Sierra Leone, SeptemberNovember, 2015

190 Notes from the Field: Verona Integron-Encoded Metallo-Beta-Lactamase-Producing CarbapenemResistant Enterobacteriaceae in a Neonatal and Adult Intensive Care Unit — Kentucky, 2015

191 Announcement

192 QuickStats

Continuing Education examination available at http://www.cdc.gov/mmwr/cme/conted_info.html\#weekly.

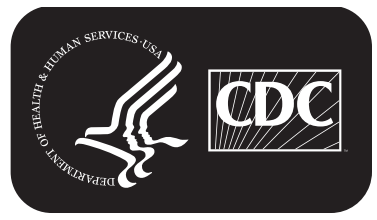

U.S. Department of Health and Human Services Centers for Disease Control and Prevention 
combined landline and cell phone data. Each year, BRFSS offers several optional modules to states on additional specific topics. In 2013, the BRFSS included an optional module with two SSB intake questions: 1) "During the past 30 days, how often did you drink regular soda or pop that contains sugar? Do not include diet soda or diet pop." and 2) "During the past 30 days, how often did you drink sugar-sweetened fruit drinks (such as Kool-Aid and lemonade), sweet tea, and sports or energy drinks (such as Gatorade and Red Bull)? Do not include $100 \%$ fruit juice, diet drinks, or artificially sweetened drinks." Respondents answered number of times per month, week, or day, and responses were converted to daily intake. To calculate daily SSB intake, daily intake frequency from both questions was summed and categorized as none, $>0$ to $<1$ (coded as $<1$ ), and $\geq 1$ time per day. $\$$ During 2013, 23 states and DC offered the SSB optional module, and 157,668 adults answered both SSB questions. Prevalence estimates are presented as percentages with $95 \%$ confidence intervals. Because age has been associated with SSB intake and age distribution varies by state, both crude and age-adjusted prevalences (standardization according to 2000 U.S. projected population) were provided for each state. Chi-square tests were used to examine whether state SSB intake varied by age, sex, race/ethnicity, employment status, or education (statistically significant at $\mathrm{p}<0.05)$. All analyses took complex survey design and sampling weight into account.

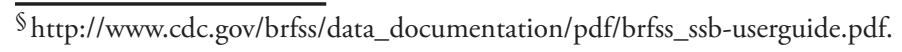

During 2013, after direct age adjustment, $30.1 \%$ of respondents reported consuming SSBs at least once per day. At least once daily SSB intake was most common among persons aged $18-24$ years (43.3\%), men (34.1\%), blacks (39.9\%), persons who reported being unemployed (34.4\%), and persons with less than a high school education (42.4\%). The lowest prevalences were reported by adults aged $\geq 55$ years $(19.1 \%)$, non-Hispanic persons of other races $(21.2 \%)$, retired persons (18.0\%), and college graduates (15.5\%). By state, the age-adjusted prevalence of daily SSB intake was highest in Mississippi (47.5\%), followed by Louisiana (45.5\%), and West Virginia (45.2\%) (Table 1). The prevalence of SSB intake of $\geq 2$ times per day ranged from a low of $8.1 \%$ in Vermont to $27.3 \%$ in Mississippi (pooled mean for 23 states and DC $=14.8 \%$ ) (data not shown).

When examined by state of residence, SSB intake was most common among younger adults (aged 18-24 years) in most states and among men in all states. Overall, the prevalence of SSB intake $\geq 1$ time per day among the youngest group of adults (persons aged 18-24 years) was 2.3 times the prevalence among the oldest age group (persons aged $\geq 55$ years), ranging from 1.6-fold higher in New York to 3.4-fold higher in New Jersey. The overall prevalence among men was approximately 1.4 times the prevalence among women, ranging from 1.1 times higher in Mississippi to 2.0 times higher in Minnesota. Similarly, when prevalence of at least once daily SSB intake among blacks and Hispanics was compared with at least once daily intake prevalence among non-Hispanic whites (whites), the prevalence among blacks was 1.5 times the prevalence among whites (ranging from 0.9 in West Virginia to

The $M M W R$ series of publications is published by the Center for Surveillance, Epidemiology, and Laboratory Services, Centers for Disease Control and Prevention (CDC), U.S. Department of Health and Human Services, Atlanta, GA 30329-4027.

Suggested citation: [Author names; first three, then et al., if more than six.] [Report title]. MMWR Morb Mortal Wkly Rep 2016;65:[inclusive page numbers].

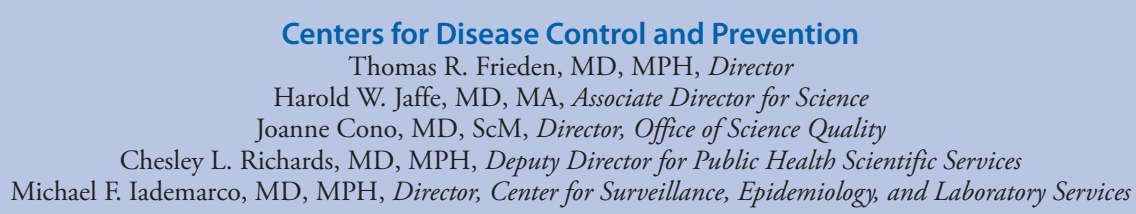

MMWR Editorial and Production Staff (Weekly)

Sonja A. Rasmussen, MD, MS, Editor-in-Chief

Charlotte K. Kent, PhD, MPH, Executive Editor Jacqueline Gindler, MD, Editor

Teresa F. Rutledge, Managing Editor

Douglas W. Weatherwax, Lead Technical Writer-Editor

Soumya Dunworth, PhD, Teresa M. Hood, MS, Technical Writer-Editors
Martha F. Boyd, Lead Visual Information Specialist Maureen A. Leahy, Julia C. Martinroe,

Stephen R. Spriggs, Moua Yang, Tong Yang, Visual Information Specialists

Quang M. Doan, MBA, Phyllis H. King, Teresa C. Moreland, Terraye M. Starr, Information Technology Specialists
MMWR Editorial Board

Timothy F. Jones, MD, Chairman

Matthew L. Boulton, MD, MPH Virginia A. Caine, MD

Katherine Lyon Daniel, PhD

Jonathan E. Fielding, MD, MPH, MBA

David W. Fleming, MD

\author{
William E. Halperin, MD, DrPH, MPH \\ King K. Holmes, MD, PhD \\ Robin Ikeda, MD, MPH \\ Rima F. Khabbaz, MD \\ Phyllis Meadows, PhD, MSN, RN \\ Jewel Mullen, MD, MPH, MPA
}

Jeff Niederdeppe, $\mathrm{PhD}$

Patricia Quinlisk, MD, MPH

Patrick L. Remington, MD, MPH Carlos Roig, MS, MA

William L. Roper, MD, MPH

William Schaffner, MD 
TABLE 1. Prevalence* of sugar-sweetened beverage ${ }^{\dagger}$ consumption among adults, by state - Behavioral Risk Factor Surveillance System, 23 states and District of Columbia, 2013

\begin{tabular}{|c|c|c|c|c|c|c|c|}
\hline \multirow[b]{2}{*}{ State } & \multirow[b]{2}{*}{ No. respondents } & \multicolumn{3}{|c|}{ Crude prevalence, $\%(95 \% \mathrm{Cl})$} & \multicolumn{3}{|c|}{ Adjusted prevalence, ${ }^{\S} \%(95 \% \mathrm{Cl})$} \\
\hline & & None & $<1$ time/day & $\geq 1$ time/day & None & $<1$ time/day & $\geq 1$ time/day \\
\hline Overall & 157,668 & $26.2(25.7-26.6)$ & $44.7(44.1-45.3)$ & $29.1(28.6-29.6)$ & $24.7(24.2-25.2)$ & $45.2(44.6-45.8)$ & $30.1(29.6-30.7)$ \\
\hline Alaska & 4,102 & $25.5(23.8-27.4)$ & $48.0(45.8-50.2)$ & $26.5(24.4-28.6)$ & $25.2(23.5-27.0)$ & $48.2(46.0-50.4)$ & $26.6(24.6-28.7)$ \\
\hline Arizona & 3,491 & $28.6(26.1-31.1)$ & $43.9(41.0-46.9)$ & $27.6(24.8-30.6)$ & $25.9(23.5-28.5)$ & $44.6(41.4-47.8)$ & $29.6(26.5-32.8)$ \\
\hline California & 5,871 & $26.8(25.4-28.3)$ & $49.4(47.6-51.1)$ & $23.8(22.3-25.4)$ & $25.8(24.4-27.2)$ & $49.9(48.1-51.7)$ & $24.3(22.8-25.9)$ \\
\hline Connecticut & 5,871 & $33.4(31.8-35.0)$ & 47.1 (45.3-48.9) & $19.5(18.1-21.1)$ & $30.8(29.3-32.5)$ & 48.6 (46.7-50.5) & $20.6(19.0-22.2)$ \\
\hline District of Columbia & 4,152 & $26.6(24.6-28.7)$ & 50.7 (48.2-53.2) & $22.7(20.4-25.1)$ & $26.4(24.5-28.4)$ & $50.2(47.7-52.8)$ & $23.4(21.1-25.8)$ \\
\hline Indiana & 4,486 & $23.1(21.7-24.5)$ & $42.0(40.1-43.9)$ & $34.9(33.1-36.9)$ & $21.5(20.2-22.9)$ & $42.3(40.3-44.3)$ & $36.2(34.2-38.2)$ \\
\hline lowa & 3,696 & $27.4(25.7-29.2)$ & 42.7 (40.6-44.8) & $29.9(27.9-32.1)$ & $25.6(24.0-27.4)$ & $42.9(40.7-45.1)$ & $31.5(29.3-33.7)$ \\
\hline Kansas & 11,121 & $26.3(25.4-27.2)$ & 43.6 (42.4-44.7) & $30.1(29.0-31.3)$ & $24.9(24.0-25.8)$ & $43.8(42.6-45.0)$ & $31.3(30.1-32.4)$ \\
\hline Kentucky & 9,818 & $21.8(20.7-23.0)$ & $36.8(35.3-38.2)$ & $41.4(39.9-42.9)$ & $20.3(19.2-21.4)$ & $36.6(35.1-38.1)$ & $43.2(41.6-44.7)$ \\
\hline Louisiana & 4,759 & $19.1(17.7-20.6)$ & $36.8(34.6-39.0)$ & $44.1(41.8-46.4)$ & $17.8(16.4-19.3)$ & $36.6(34.4-39.0)$ & $45.5(43.1-48.0)$ \\
\hline Maryland & 11,759 & $28.1(27.0-29.3)$ & $44.4(43.0-45.8)$ & $27.5(26.1-28.9)$ & $26.2(25.1-27.4)$ & $45.3(43.8-46.8)$ & $28.5(27.0-30.0)$ \\
\hline Minnesota & 12,704 & $30.6(29.2-32.1)$ & $48.1(46.5-49.8)$ & $21.2(19.9-22.6)$ & $28.7(27.4-30.1)$ & $49.0(47.3-50.6)$ & $22.3(21.0-23.8)$ \\
\hline Mississippi & 6,692 & $16.4(15.3-17.5)$ & $37.4(35.7-39.1)$ & $46.2(44.5-48.1)$ & $15.4(14.3-16.5)$ & 37.1 (35.3-38.9) & $47.5(45.7-49.4)$ \\
\hline Nebraska & 7,822 & $24.4(23.0-25.8)$ & 47.1 (45.4-48.9) & $28.5(26.8-30.3)$ & $23.2(21.8-24.6)$ & $47.0(45.2-48.9)$ & $29.8(28.0-31.7)$ \\
\hline New Jersey & 3,842 & $34.0(31.9-36.2)$ & $43.0(40.6-45.4)$ & $23.0(20.9-25.1)$ & $32.0(30.1-34.1)$ & $43.8(41.3-46.3)$ & $24.2(22.1-26.4)$ \\
\hline New York & 3,751 & $30.2(28.5-32.1)$ & $47.3(45.2-49.4)$ & $22.5(20.7-24.3)$ & $29.1(27.3-30.8)$ & $47.9(45.8-50.0)$ & $23.0(21.2-25.0)$ \\
\hline North Carolina & 3,926 & $19.6(18.1-21.2)$ & $40.1(38.0-42.3)$ & $40.3(38.1-42.5)$ & $18.3(16.9-19.9)$ & $40.2(38.0-42.4)$ & $41.5(39.3-43.7)$ \\
\hline Ohio & 7,316 & $25.4(24.1-26.8)$ & $43.4(41.7-45.1)$ & $31.2(29.6-32.9)$ & $23.7(22.4-25.0)$ & $43.5(41.7-45.4)$ & $32.8(31.1-34.6)$ \\
\hline Oklahoma & 3,638 & $19.0(17.5-20.5)$ & $37.9(35.9-40.0)$ & $43.1(40.9-45.3)$ & $17.9(16.4-19.4)$ & $37.6(35.5-39.7)$ & $44.6(42.4-46.8)$ \\
\hline South Carolina & 9,590 & 19.8 (18.8-20.9) & 39.8 (38.4-41.2) & $40.4(39.0-41.9)$ & $18.0(17.1-19.1)$ & 39.9 (38.4-41.4) & $42.1(40.6-43.6)$ \\
\hline Utah & 11,428 & $20.9(20.1-21.8)$ & $51.6(50.4-52.8)$ & $27.5(26.4-28.6)$ & $21.8(20.9-22.6)$ & $51.2(50.0-52.4)$ & $27.0(26.0-28.1)$ \\
\hline Vermont & 5,784 & $35.8(34.3-37.4)$ & $47.5(45.8-49.2)$ & $16.7(15.4-18.1)$ & $32.7(31.2-34.2)$ & $49.3(47.5-51.1)$ & $18.0(16.5-19.6)$ \\
\hline West Virginia & 5,630 & $22.4(21.2-23.6)$ & $35.9(34.4-37.4)$ & $41.8(40.2-43.4)$ & $19.7(18.6-20.9)$ & $35.1(33.5-36.7)$ & $45.2(43.5-46.9)$ \\
\hline Wisconsin & 5,500 & $26.4(24.7-28.2)$ & $46.9(44.8-49.0)$ & $26.7(24.8-28.7)$ & $24.0(22.4-25.8)$ & $47.4(45.2-49.7)$ & $28.5(26.5-30.6)$ \\
\hline
\end{tabular}

Abbreviation: $\mathrm{Cl}=$ confidence interval.

* Weighted percentages might not sum to $100 \%$ because of rounding.

+ Included regular soda, fruit drinks, sweet tea, and sports or energy drinks.

$\S$ Age standardization according to 2000 U.S. projected population.

4.9 in DC), and among Hispanics, was 1.4 times the prevalence among whites (ranging from 0.7 in Vermont to 2.2 in California). Overall, the prevalence of at least once daily SSB consumption among adults with less than a high school education was 2.7 times the prevalence among college graduates, ranging from 1.5 times higher in Mississippi to 5.4 times higher in DC.

The states with the highest prevalences of at least once daily consumption of SSBs among persons aged 18-24 years were Oklahoma (66.4\%) and West Virginia (63.8\%). The highest prevalences among men were in Louisiana (50.6\%) and Mississippi (48.7\%). At least once daily SSB intake was most prevalent among blacks in approximately half of states; states with the highest prevalence among blacks were Louisiana $(50.7 \%)$ and Iowa (49.0\%) (Table 2). Among persons who were unemployed, the highest prevalences of consuming SSBs $\geq 1$ time per day were reported in Mississippi (49.5\%) and Louisiana (48.4\%). The states with the highest prevalence among persons with less than a high school education were Louisiana (60.0\%) and South Carolina (55.6\%) (Table 3).

\section{Discussion}

The frequency of daily SSB intake remains high in some states and within certain populations. During 2013, approximately one in three adults reported consuming SSBs at least once daily in DC and the 23 states surveyed. Adults in Louisiana, Mississippi, and West Virginia reported the highest prevalence of at least once daily SSB intake. Daily SSB intake was most frequently reported among persons aged 18-24 years, men, blacks, adults who were not employed, and persons with less than a high school education.

The prevalence of daily SSB intake in this study was somewhat lower than that reported by the 2009-2010 NHANES data, which found that $50.6 \%$ of U.S. adults reported consuming at least one SSB on a given day (3). Potential reasons for this discrepancy might be accounted for by differences in modes of survey administration, methods of collecting dietary intake data, survey year, representativeness, and usual intake assessment. NHANES is an in-person and phone follow-up survey using 24-hour dietary recalls with USDA's Automated Multiple-Pass Method that captures all forms of SSBs. In contrast, the BRFSS is a telephone survey using a short dietary screener about usual intake during the past 30 days.

As has been reported in other studies that used National Health Interview Survey and BRFSS $(4,6)$ data, the prevalence of at least once daily SSB intake in this analysis was higher in southern states. Higher SSB intake frequency in certain states could result, in part, from variations in beverage retail 
TABLE 2. Crude prevalence* of sugar-sweetened beverage ${ }^{\dagger}$ consumption $\geq 1$ time/day among adults, by age group, sex, race/ethnicity, and state - Behavioral Risk Factor Surveillance System, 23 states and District of Columbia, 2013

\begin{tabular}{|c|c|c|c|c|c|c|c|c|c|c|c|}
\hline \multirow[b]{3}{*}{ State } & \multirow[b]{3}{*}{$\begin{array}{l}\text { No. } \\
\text { respondents }\end{array}$} & \multicolumn{10}{|c|}{ Crude prevalence of sugar-sweetened beverage consumption $\geq 1$ time/day, $\%(95 \% \mathrm{Cl})$} \\
\hline & & \multicolumn{4}{|c|}{ Age group $(y r s)^{\S}$} & \multicolumn{2}{|c|}{ Sex $x^{\S}$} & \multicolumn{4}{|c|}{ Race/Ethnicity§ } \\
\hline & & $18-24$ & $25-34$ & $35-54$ & $\geq 55$ & Male & Female & $\begin{array}{c}\text { White, } \\
\text { non-Hispanic }\end{array}$ & $\begin{array}{c}\text { Black, } \\
\text { non-Hispanic }\end{array}$ & Hispanic & $\begin{array}{c}\text { Other, } \\
\text { non-Hispanic }\end{array}$ \\
\hline verall? & 157,668 & $\begin{array}{r}43.3 \\
(41.2-45.5)\end{array}$ & $\begin{array}{r}38.2 \\
(36.7-39.7)\end{array}$ & $\begin{array}{r}30.1 \\
(29.3-31.0)\end{array}$ & $\begin{array}{r}19.1 \\
(18.5-19.7)\end{array}$ & $\begin{array}{r}34.1 \\
(33.3-35.0)\end{array}$ & $\begin{array}{r}24.4 \\
(23.8-25.0)\end{array}$ & $\begin{array}{r}26.7 \\
(26.2-27.2)\end{array}$ & $\begin{array}{r}39.9 \\
(38.1-41.7)\end{array}$ & $\begin{array}{r}36.3 \\
(34.4-38.3)\end{array}$ & $\begin{array}{r}21.2 \\
(19.2-23.4)\end{array}$ \\
\hline Alaska & 4,102 & $\begin{array}{r}45.9 \\
(38.7-53.3)\end{array}$ & $\begin{array}{r}31.3 \\
(26.3-36.7)\end{array}$ & $\begin{array}{r}25.1 \\
(22.0-28.4)\end{array}$ & $\begin{array}{r}17.1 \\
(14.5-20.1)\end{array}$ & $\begin{array}{r}31.2 \\
(28.3-34.3)\end{array}$ & $\begin{array}{r}21.5 \\
(18.8-24.4)\end{array}$ & $\begin{array}{r}21.1 \\
(19.0-23.3)\end{array}$ & $\begin{array}{r}31.5 \\
(18.7-47.8)\end{array}$ & $\begin{array}{r}26.4 \\
(16.3-39.8)\end{array}$ & $\begin{array}{r}41.4 \\
(36.5-46.6)\end{array}$ \\
\hline Arizona & 3,491 & $\begin{array}{r}48.8 \\
(38.1-59.7)\end{array}$ & $\begin{array}{r}41.5 \\
(32.4-51.1)\end{array}$ & $\begin{array}{r}27.9 \\
(23.2-33.0)\end{array}$ & $\begin{array}{r}15.7 \\
(13.0-18.9)\end{array}$ & $\begin{array}{r}34.4 \\
(29.9-39.2)\end{array}$ & $\begin{array}{r}21.6 \\
(18.3-25.3)\end{array}$ & $\begin{array}{r}21.5 \\
(18.9-24.5)\end{array}$ & $\begin{array}{r}32.9 \\
(18.3-51.8)\end{array}$ & $\begin{array}{r}39.4 \\
(32.0-47.3)\end{array}$ & $\begin{array}{r}39.1 \\
(28.0-51.4)\end{array}$ \\
\hline alifornia & 5,871 & $\begin{array}{r}36.4 \\
(30.8-42.4)\end{array}$ & $\begin{array}{r}31.5 \\
(27.5-35.9)\end{array}$ & $\begin{array}{r}23.9 \\
(21.5-26.4)\end{array}$ & $\begin{array}{r}14.9 \\
(13.1-17.0)\end{array}$ & $\begin{array}{r}28.2 \\
(25.8-30.7)\end{array}$ & $\begin{array}{r}19.4 \\
(17.6-21.5)\end{array}$ & $\begin{array}{r}16.3 \\
(14.6-18.1)\end{array}$ & $\begin{array}{r}33.5 \\
(25.3-43.3)\end{array}$ & $\begin{array}{r}36.0 \\
(33.1-39.0)\end{array}$ & $\begin{array}{r}14.4 \\
(11.2-18.4)\end{array}$ \\
\hline Connecticut & 5,871 & $\begin{array}{r}30.4 \\
(23.9-37.8)\end{array}$ & $\begin{array}{r}29.1 \\
(24.5-34.2)\end{array}$ & $\begin{array}{r}18.0 \\
(15.8-20.4)\end{array}$ & $\begin{array}{r}14.3 \\
(12.7-16.0)\end{array}$ & $\begin{array}{r}24.2 \\
(22.0-26.6)\end{array}$ & $\begin{array}{r}15.3 \\
(13.5-17.3)\end{array}$ & $\begin{array}{r}16.6 \\
(15.1-18.2)\end{array}$ & $\begin{array}{r}30.3 \\
(24.8-36.4)\end{array}$ & $\begin{array}{r}31.1 \\
(25.4-37.4)\end{array}$ & $\begin{array}{r}18.4 \\
(12.0-27.0)\end{array}$ \\
\hline $\begin{array}{l}\text { District of } \\
\text { Columbia }\end{array}$ & 4,152 & $\begin{array}{r}43.0 \\
(32.1-54.7)\end{array}$ & $\begin{array}{r}20.8 \\
(15.9-26.7)\end{array}$ & $\begin{array}{r}20.3 \\
(17.0-24.0)\end{array}$ & $\begin{array}{r}20.5 \\
(18.1-23.1)\end{array}$ & $\begin{array}{r}24.7 \\
(21.1-28.6)\end{array}$ & $\begin{array}{r}20.9 \\
(18.2-23.9)\end{array}$ & $\begin{array}{r}7.5 \\
(5.6-10.0)\end{array}$ & $\begin{array}{r}37.2 \\
(33.4-41.1)\end{array}$ & $\begin{array}{r}16.5 \\
(9.6-26.9)\end{array}$ & $\begin{array}{r}12.7 \\
(7.6-20.3)\end{array}$ \\
\hline Indiana & 4,486 & $\begin{array}{r}45.2 \\
(37.7-52.9)\end{array}$ & $\begin{array}{r}45.4 \\
(39.8-51.1)\end{array}$ & $\begin{array}{r}37.5 \\
(34.4-40.7)\end{array}$ & $\begin{array}{r}24.6 \\
(22.5-26.7)\end{array}$ & $\begin{array}{r}39.3 \\
(36.5-42.3)\end{array}$ & $\begin{array}{r}30.9 \\
(28.5-33.4)\end{array}$ & $\begin{array}{r}33.5 \\
(31.5-35.5)\end{array}$ & $\begin{array}{r}39.7 \\
(32.4-47.6)\end{array}$ & $\begin{array}{r}43.1 \\
(33.5-53.3)\end{array}$ & $\begin{array}{r}45.6 \\
(33.4-58.3)\end{array}$ \\
\hline a & 3,696 & $\begin{array}{r}52.5 \\
(44.1-60.6)\end{array}$ & $\begin{array}{r}40.2 \\
(34.2-46.5)\end{array}$ & $\begin{array}{r}31.7 \\
(28.3-35.4)\end{array}$ & $\begin{array}{r}16.3 \\
(14.4-18.3)\end{array}$ & $\begin{array}{r}38.2 \\
(34.9-41.5)\end{array}$ & $\begin{array}{r}22.0 \\
(19.5-24.8)\end{array}$ & $\begin{array}{r}28.3 \\
(26.2-30.4)\end{array}$ & $\begin{array}{r}49.0 \\
(31.0-67.2)\end{array}$ & $\begin{array}{r}37.1 \\
(25.5-50.4)\end{array}$ & $\begin{array}{r}63.0 \\
(43.9-78.7)\end{array}$ \\
\hline ansas. & 11,121 & $\begin{array}{r}45.3 \\
(41.0-49.6)\end{array}$ & $\begin{array}{r}39.4 \\
(36.2-42.7)\end{array}$ & $\begin{array}{r}32.1 \\
(30.2-33.9)\end{array}$ & $\begin{array}{r}18.7 \\
(17.6-19.9)\end{array}$ & $\begin{array}{r}36.0 \\
(34.3-37.7)\end{array}$ & $\begin{array}{r}24.6 \\
(23.2-26.0)\end{array}$ & $\begin{array}{r}28.8 \\
(27.7-30.0)\end{array}$ & $\begin{array}{r}32.0 \\
(26.3-38.2)\end{array}$ & $\begin{array}{r}42.5 \\
(37.7-47.5)\end{array}$ & $\begin{array}{r}28.2 \\
(23.1-34.1)\end{array}$ \\
\hline entucky & 9,818 & $\begin{array}{r}56.8 \\
(51.2-62.3)\end{array}$ & $\begin{array}{r}55.5 \\
(51.0-59.9)\end{array}$ & $\begin{array}{r}44.1 \\
(41.5-46.7)\end{array}$ & $\begin{array}{r}27.9 \\
(26.1-29.8)\end{array}$ & $\begin{array}{r}46.8 \\
(44.4-49.1)\end{array}$ & $\begin{array}{r}36.5 \\
(34.5-38.5)\end{array}$ & $\begin{array}{r}41.4 \\
(39.8-42.9)\end{array}$ & $\begin{array}{r}44.2 \\
(36.7-52.0)\end{array}$ & $\begin{array}{r}36.3 \\
(24.1-50.7)\end{array}$ & $\begin{array}{r}34.6 \\
(26.3-44.1)\end{array}$ \\
\hline uisiana & 4,759 & $\begin{array}{r}58.2 \\
(49.4-66.6)\end{array}$ & $\begin{array}{r}55.7 \\
(48.4-62.7)\end{array}$ & $\begin{array}{r}47.0 \\
(43.1-51.0)\end{array}$ & $\begin{array}{r}31.3 \\
(28.9-33.7)\end{array}$ & $\begin{array}{r}50.6 \\
(46.9-54.4)\end{array}$ & $\begin{array}{r}38.3 \\
(35.5-41.2)\end{array}$ & $\begin{array}{r}41.0 \\
(38.3-43.8)\end{array}$ & $\begin{array}{r}50.7 \\
(46.1-55.2)\end{array}$ & $\begin{array}{r}41.2 \\
(26.0-58.3)\end{array}$ & $\begin{array}{r}41.2 \\
(27.5-56.5)\end{array}$ \\
\hline nd & 9 & $\begin{array}{r}40.8 \\
(34.7-47.2)\end{array}$ & $\begin{array}{r}32.5 \\
(28.6-36.8)\end{array}$ & $\begin{array}{r}28.7 \\
(26.6-30.9)\end{array}$ & $\begin{array}{r}20.0 \\
(18.6-21.5)\end{array}$ & $\begin{array}{r}32.2 \\
(30.0-34.5)\end{array}$ & $\begin{array}{r}23.3 \\
(21.7-25.0)\end{array}$ & $\begin{array}{r}24.6 \\
(23.1-26.3)\end{array}$ & $\begin{array}{r}34.1 \\
(31.1-37.3)\end{array}$ & $\begin{array}{r}29.8 \\
(24.2-36.2)\end{array}$ & $\begin{array}{r}22.9 \\
(18.2-28.5)\end{array}$ \\
\hline innesota & 12,704 & $\begin{array}{r}33.1 \\
(28.2-38.4)\end{array}$ & $\begin{array}{r}33.8 \\
(30.2-37.7)\end{array}$ & $\begin{array}{r}21.1 \\
(18.9-23.4)\end{array}$ & $\begin{array}{r}11.9 \\
(10.3-13.8)\end{array}$ & $\begin{array}{r}28.3 \\
(26.2-30.6)\end{array}$ & $\begin{array}{r}14.5 \\
(13.0-16.2)\end{array}$ & $\begin{array}{r}20.0 \\
(18.6-21.5)\end{array}$ & $\begin{array}{r}28.9 \\
(21.1-38.1)\end{array}$ & $\begin{array}{r}38.9 \\
(30.4-48.1)\end{array}$ & $\begin{array}{r}20.5 \\
(15.2-27.2)\end{array}$ \\
\hline Mississippi & 6,692 & $\begin{array}{r}62.5 \\
(55.9-68.8)\end{array}$ & $\begin{array}{r}57.3 \\
(52.1-62.2)\end{array}$ & $\begin{array}{r}48.1 \\
(45.1-51.2)\end{array}$ & $\begin{array}{r}33.8 \\
(31.8-36.0)\end{array}$ & $\begin{array}{r}48.7 \\
(45.8-51.6)\end{array}$ & $\begin{array}{r}44.1 \\
(41.9-46.3)\end{array}$ & $\begin{array}{r}45.0 \\
(42.7-47.2)\end{array}$ & $\begin{array}{r}48.7 \\
(45.5-51.9)\end{array}$ & $\begin{array}{r}54.9 \\
(39.7-69.3)\end{array}$ & $\begin{array}{r}39.2 \\
(27.3-52.6)\end{array}$ \\
\hline ebraska & 7,822 & $\begin{array}{r}42.0 \\
(35.6-48.7)\end{array}$ & $\begin{array}{r}40.7 \\
(35.7-45.8)\end{array}$ & $\begin{array}{r}31.3 \\
(28.3-34.5)\end{array}$ & $\begin{array}{r}15.4 \\
(13.8-17.1)\end{array}$ & $\begin{array}{r}35.8 \\
(33.1-38.5)\end{array}$ & $\begin{array}{r}21.7 \\
(19.7-24.0)\end{array}$ & $\begin{array}{r}26.4 \\
(24.7-28.2)\end{array}$ & $\begin{array}{r}36.7 \\
(24.7-50.7)\end{array}$ & $\begin{array}{r}48.7 \\
(40.1-57.4)\end{array}$ & $\begin{array}{r}31.3 \\
(22.1-42.3)\end{array}$ \\
\hline tacy & 3,842 & $\begin{array}{r}46.2 \\
(36.5-56.2)\end{array}$ & $\begin{array}{r}28.6 \\
(23.2-34.7)\end{array}$ & $\begin{array}{r}22.6 \\
(19.6-25.9)\end{array}$ & $\begin{array}{r}13.8 \\
(11.6-16.2)\end{array}$ & $\begin{array}{r}28.0 \\
(24.8-31.5)\end{array}$ & $\begin{array}{r}18.2 \\
(15.8-20.9)\end{array}$ & $\begin{array}{r}19.9 \\
(17.6-22.5)\end{array}$ & $\begin{array}{r}38.3 \\
(31.8-45.4)\end{array}$ & $\begin{array}{r}29.8 \\
(23.9-36.3)\end{array}$ & $\begin{array}{r}11.0 \\
(6.8-17.3)\end{array}$ \\
\hline New & 3,751 & $\begin{array}{r}25.1 \\
(18.7-32.9)\end{array}$ & $\begin{array}{r}29.6 \\
(24.9-34.7)\end{array}$ & $\begin{array}{r}24.3 \\
(21.3-27.6)\end{array}$ & $\begin{array}{r}16.2 \\
(14.2-18.5)\end{array}$ & $\begin{array}{r}27.1 \\
(24.3-30.1)\end{array}$ & $\begin{array}{r}18.2 \\
(16.1-20.4)\end{array}$ & $\begin{array}{r}17.9 \\
(15.9-20.1)\end{array}$ & $\begin{array}{r}33.7 \\
(28.1-39.8)\end{array}$ & $\begin{array}{r}32.9 \\
(27.9-38.3)\end{array}$ & $\begin{array}{r}17.1 \\
(11.9-24.0)\end{array}$ \\
\hline $\begin{array}{l}\text { North } \\
\text { Carolina }\end{array}$ & 3,926 & $\begin{array}{r}61.7 \\
(53.3-69.4)\end{array}$ & $\begin{array}{r}46.5 \\
(40.5-52.5)\end{array}$ & $\begin{array}{r}42.8 \\
(39.3-46.5)\end{array}$ & $\begin{array}{r}27.4 \\
(24.8-30.1)\end{array}$ & $\begin{array}{r}44.2 \\
(40.9-47.6)\end{array}$ & $\begin{array}{r}36.6 \\
(33.8-39.5)\end{array}$ & $\begin{array}{r}38.5 \\
(35.9-41.1)\end{array}$ & $\begin{array}{r}46.0 \\
(41.0-51.1)\end{array}$ & $\begin{array}{r}49.9 \\
(41.9-57.8)\end{array}$ & $\begin{array}{r}26.4 \\
(17.7-37.5)\end{array}$ \\
\hline Ohio & 7,316 & $\begin{array}{r}49.4 \\
(42.5-56.3)\end{array}$ & $\begin{array}{r}44.1 \\
(39.1-49.2)\end{array}$ & $\begin{array}{r}31.9 \\
(29.3-34.6)\end{array}$ & $\begin{array}{r}19.5 \\
(17.8-21.3)\end{array}$ & $\begin{array}{r}37.1 \\
(34.6-39.8)\end{array}$ & $\begin{array}{r}25.7 \\
(23.7-27.8)\end{array}$ & $\begin{array}{r}29.9 \\
(28.2-31.6)\end{array}$ & $\begin{array}{r}41.6 \\
(35.8-47.6)\end{array}$ & $\begin{array}{r}38.1 \\
(25.0-53.1)\end{array}$ & $\begin{array}{r}30.3 \\
(21.6-40.7)\end{array}$ \\
\hline Oklahoma & 3,638 & $\begin{array}{r}66.4 \\
(58.0-73.9)\end{array}$ & $\begin{array}{r}57.4 \\
(51.4-63.1)\end{array}$ & $\begin{array}{r}45.1 \\
(41.4-48.8)\end{array}$ & $\begin{array}{r}25.9 \\
(23.6-28.4)\end{array}$ & $\begin{array}{r}45.8 \\
(42.5-49.2)\end{array}$ & $\begin{array}{r}40.5 \\
(37.7-43.3)\end{array}$ & $\begin{array}{r}40.9 \\
(38.4-43.5)\end{array}$ & $\begin{array}{r}39.7 \\
(31.4-48.7)\end{array}$ & $\begin{array}{r}53.8 \\
(45.0-62.5)\end{array}$ & $\begin{array}{r}50.5 \\
(44.5-56.4)\end{array}$ \\
\hline $\begin{array}{l}\text { South } \\
\text { Carolina }\end{array}$ & 90 & $\begin{array}{r}58.7 \\
(53.0-64.1)\end{array}$ & $\begin{array}{r}51.9 \\
(47.8-56.0)\end{array}$ & $\begin{array}{r}41.4 \\
(38.9-43.9)\end{array}$ & $\begin{array}{r}29.4 \\
(27.6-31.2)\end{array}$ & $\begin{array}{r}46.4 \\
(44.2-48.6)\end{array}$ & $\begin{array}{r}35.1 \\
(33.2-37.0)\end{array}$ & $\begin{array}{r}38.6 \\
(36.9-40.4)\end{array}$ & $\begin{array}{r}45.1 \\
(42.2-48.1)\end{array}$ & $\begin{array}{r}38.5 \\
(28.4-49.6)\end{array}$ & $\begin{array}{r}40.7 \\
(33.0-48.8)\end{array}$ \\
\hline Utah & 11,428 & $\begin{array}{r}32.6 \\
(29.1-36.3)\end{array}$ & $\begin{array}{r}35.4 \\
(32.7-38.2)\end{array}$ & $\begin{array}{r}27.2 \\
(25.4-29.0)\end{array}$ & $\begin{array}{r}19.0 \\
(17.6-20.5)\end{array}$ & $\begin{array}{r}34.3 \\
(32.6-36.0)\end{array}$ & $\begin{array}{r}20.9 \\
(19.5-22.3)\end{array}$ & $\begin{array}{r}25.3 \\
(24.2-26.4)\end{array}$ & $\begin{array}{r}36.4 \\
(21.5-54.5)\end{array}$ & $\begin{array}{r}42.1 \\
(37.8-46.6)\end{array}$ & $\begin{array}{r}28.7 \\
(23.1-35.1)\end{array}$ \\
\hline Vermont & 5,784 & $\begin{array}{r}25.5 \\
(19.5-32.6)\end{array}$ & $\begin{array}{r}24.5 \\
(20.2-29.4)\end{array}$ & $\begin{array}{r}18.0 \\
(15.8-20.4)\end{array}$ & $\begin{array}{r}10.6 \\
(9.4-12.0)\end{array}$ & $\begin{array}{r}21.7 \\
(19.5-24.1)\end{array}$ & $\begin{array}{r}12.2 \\
(10.7-13.9)\end{array}$ & $\begin{array}{r}16.3 \\
(15.0-17.8)\end{array}$ & $\begin{array}{r}31.9 \\
(11.5-63.0)\end{array}$ & $\begin{array}{r}11.2 \\
(4.1-27.0)\end{array}$ & $\begin{array}{r}29.7 \\
(21.7-39.3)\end{array}$ \\
\hline West Virginia & 5,630 & $\begin{array}{r}63.8 \\
(57.4-69.9)\end{array}$ & $\begin{array}{r}56.5 \\
(51.8-61.2)\end{array}$ & $\begin{array}{r}48.0 \\
(45.4-50.7)\end{array}$ & $\begin{array}{r}25.7 \\
(24.0-27.6)\end{array}$ & $\begin{array}{r}47.3 \\
(44.9-49.8)\end{array}$ & $\begin{array}{r}36.5 \\
(34.5-38.5)\end{array}$ & $\begin{array}{r}42.0 \\
(40.4-43.6)\end{array}$ & $\begin{array}{r}37.1 \\
(26.4-49.2)\end{array}$ & $\begin{array}{r}40.0 \\
(25.1-57.0)\end{array}$ & $\begin{array}{r}40.3 \\
(30.6-50.8)\end{array}$ \\
\hline Wisconsin & 5,500 & $\begin{array}{r}44.1 \\
(36.8-51.7)\end{array}$ & $\begin{array}{r}41.6 \\
(35.6-47.9)\end{array}$ & $\begin{array}{r}27.8 \\
(24.7-31.2)\end{array}$ & $\begin{array}{r}14.2 \\
(12.3-16.4)\end{array}$ & $\begin{array}{r}33.1 \\
(30.2-36.2)\end{array}$ & $\begin{array}{r}20.4 \\
(17.9-23.0)\end{array}$ & $\begin{array}{r}24.9 \\
(23.0-26.9)\end{array}$ & $\begin{array}{r}48.2 \\
(36.4-60.2)\end{array}$ & $\begin{array}{r}40.4 \\
(27.3-55.0)\end{array}$ & $\begin{array}{r}25.2 \\
(17.1-35.6)\end{array}$ \\
\hline
\end{tabular}

Abbreviation: $\mathrm{Cl}=$ confidence interval.

* Weighted percentages might not sum to $100 \%$ because of rounding.

+ Includes regular soda, fruit drinks, sweet tea, and sports or energy drinks.

$\S$ Values for all states were significantly different at $\mathrm{p}<0.05$ by chi-square test except for Virginia, where differences in SSB intake by race/ethnicity were not statistically significant.

" Missing data: $1.2 \%$ for race/ethnicity. 
Morbidity and Mortality Weekly Report

TABLE 3. Crude prevalence* of sugar-sweetened beverage ${ }^{\dagger}$ consumption $\geq 1$ time/day among adults, by employment status, education, and state - Behavioral Risk Factor Surveillance System, 23 states and District of Columbia, 2013

\begin{tabular}{|c|c|c|c|c|c|c|c|c|}
\hline \multirow[b]{3}{*}{ State } & \multirow[b]{3}{*}{ No. respondents } & \multicolumn{7}{|c|}{ Crude prevalence of sugar-sweetened beverage consumption $\geq 1$ time/day $(95 \% \mathrm{Cl})$} \\
\hline & & \multicolumn{3}{|c|}{ Employment status $\S$} & \multicolumn{4}{|c|}{ Education $\$$} \\
\hline & & Employed & Not employed & Retired & $<$ High school & High school & Some college & College graduate \\
\hline Overallq & 157,668 & $30.0(29.3-30.7)$ & $4.4(33.2-35.5)$ & 18.0(17.2-18.9) & $42.4(40.6-44.3)$ & $35.8(34.8-36.8)$ & $28.5(27.6-29.5)$ & $15.5(14.9-16.2)$ \\
\hline Alaska & 4,102 & $26.2(23.6-28.9)$ & $32.1(27.8-36.8)$ & $16.0(12.6-20.2)$ & $47.1(37.8-56.5)$ & $34.9(31.1-38.9)$ & $24.2(21.0-27.8)$ & $12.9(10.5$ \\
\hline Arizona & 3,491 & $.0(28.5-37.8)$ & $28.7(23.5-34.5)$ & $13.8(10.8-17.5)$ & $40.4(30.9-50.7)$ & $36.5(30.7-42.7)$ & $24.4(19.9-29.4)$ & $14.6(11.6-18.3)$ \\
\hline California & 5,871 & $22.9(20.9-25.1)$ & $30.2(27.1-33.4)$ & $15.0(12.2-18.2)$ & $38.5(34.2-43.0)$ & $29.9(26.5-33.7)$ & $21.4(18.8-24.2)$ & $11.5(9.8$ \\
\hline Connecticut & 5,871 & $18.9(17.1-20.9)$ & $24.3(20.8-28.2)$ & $15.0(12.7-17.7)$ & $27.8(22.4-33.9)$ & $26.9(23.7-30.3)$ & $19.9(17.2-23.0)$ & $10.2(8.7-12.0)$ \\
\hline $\begin{array}{l}\text { District of } \\
\text { Columbia }\end{array}$ & 4,152 & $18.5(15.7-21.7)$ & $34.6(29.5-40.1)$ & $18.5(15.3-22.1)$ & $45.6(36.4-55.2)$ & $39.0(33.1-45.2)$ & $28.9(23.4-35.0)$ & $8.4(7.0-10.1)$ \\
\hline Indiana & & 5) & 40 & 3) & 0) & 2.9) & 6.6) & 9) \\
\hline lowa & & $34.2(3$ & $33.1(2$ & $12.0(9.9-14.6)$ & $49.2(40.0-58.5)$ & $34.6(31.1-38.3)$ & $28.8(25.2-32.6)$ & 20.6) \\
\hline & 11 & $32.4(31.0-33.9)$ & $35.3(32.6-38.0)$ & $15.4(14.0-17.0)$ & $45.2(40.9-49.7)$ & $37.0(34.8-39.2)$ & $30.6(28.6-32.6)$ & $17.2(15.8-18.7)$ \\
\hline & & 5) & 46.1 & $27.3(24$ & $53.4(4$ & $-49.6)$ & $39.7(37.1-42.4)$ & 6.2) \\
\hline ina & 4,759 & $0.3)$ & .8-53.0) & $29.3(26.4-32.5)$ & $60.0(53.3-66.5)$ & $48.6(44.8-52.5)$ & $40.7(36.6-44.9)$ & 9.8) \\
\hline Maryland & 11,759 & 28.0 & $3.3-34.9)$ & $20.8(18.7-23.1)$ & $40.7(35.2-46.5)$ & $36.9(34.1-39.9)$ & $27.2(24.7-29.9)$ & 15.5( \\
\hline Minnesota & 12,704 & $23.5(21.8-25.3)$ & $25.0(21.5-28.9)$ & $8.9(6.8-11.7)$ & $30.6(24.6-37.3)$ & $27.1(24.2-30.1)$ & $21.9(19.6-24.4)$ & 12.7( \\
\hline Mississippi & 6,692 & $49.7(47.1-52.3)$ & $49.5(46.1-52.9)$ & $29.7(27.0-32.5)$ & $51.1(46.6-55.6)$ & $49.2(46.0-52.4)$ & $47.2(43.8-50.6)$ & $34.9(31.9-38.0)$ \\
\hline Nebraska & 7,822 & $31.1(28.9-33.4)$ & $30.5(26.3-35.1)$ & $14.9(12.5-17.6)$ & $52.3(45.0-59.5)$ & $37.0(33.8-40.3)$ & $25.9(23.2-28.8)$ & $14.3(12.2-16.6)$ \\
\hline New Jersey & 3,842 & $22.7(20.1-25.6)$ & $30.1(25.3-35.3)$ & $13.3(10.4-17.0)$ & $40.0(32.2-48.3)$ & $29.5(25.4-33.9)$ & $22.2(18.6-26.4)$ & $11.5(9.5-13.8)$ \\
\hline New York & 3,751 & $23.1(20.7-25.7)$ & $24.6(21.0-28.7)$ & $16.1(13.4-19.3)$ & $31.3(25.2-38.1)$ & $26.1(22.4-30.2)$ & $24.2(21.0-27.8)$ & $13.6(11.6-15.9)$ \\
\hline North Carolina & 3,926 & $42.1(39.2-45.1)$ & $46.0(41.5-50.7)$ & $25.9(22.4-29.8)$ & $52.1(46.2-57.9)$ & $47.2(43.0-51.3)$ & $41.5(37.4-45.7)$ & $23.5(20.4-26.9)$ \\
\hline Ohio & 7,316 & $32.0(29.9-34.3)$ & $39.0(35.3-42.8)$ & $18.1(15.7-20.8)$ & $44.4(38.0-50.9)$ & $36.6(33.8-39.5)$ & $31.8(28.9-34.9)$ & $16.0(14.0-18.2)$ \\
\hline Oklahoma & 3,638 & $46.5(43.5-49.6)$ & $48.2(43.8-52.6)$ & $21.9(19.0-25.1)$ & $51.5(44.5-58.3)$ & $50.8(46.9-54.6)$ & $43.5(39.7-47.4)$ & $24.9(21.8-28.3)$ \\
\hline South Carolina & 9,590 & $42.0(40.0-44.1)$ & $46.5(43.6-49.4)$ & $26.2(23.8-28.6)$ & $55.6(51.3-59.7)$ & $46.5(43.9-49.1)$ & $39.1(36.5-41.8)$ & $23.5(21.4-25.7)$ \\
\hline Utah & 11,428 & $30.3(28.9-31.8)$ & $25.6(23.3-27.9)$ & $17.4(15.5-19.5)$ & $48.5(43.1-53.8)$ & $36.4(34.2-38.6)$ & $25.0(23.3-26.7)$ & $15.1(13.8-16.5)$ \\
\hline Vermont & 5,784 & $16.7(15.0-18.6)$ & $21.5(18.0-25.5)$ & $11.2(9.4-13.4)$ & $32.9(26.0-40.6)$ & $21.5(19.0-24.2)$ & $16.9(14.3-19.8)$ & $7.8(6.6-9.3)$ \\
\hline West Virginia & 5,630 & $46.1(43.7-48.4)$ & $47.7(44.7-50.8)$ & $22.3(20.0-24.9)$ & $47.5(43.1-51.8)$ & $46.6(44.1-49.1)$ & $40.7(37.6-43.9)$ & $26.1(23.4-29.0)$ \\
\hline Wisconsin & 5,500 & $29.7(27.1-32.3)$ & $30.8(26.1-35.8)$ & 12.5 (10.0-15.5) & $35.5(27.5-44.5)$ & $32.6(29.2-36.2)$ & $27.6(24.2-31.3)$ & $13.6(11.5-16.1)$ \\
\hline
\end{tabular}

Abbreviation: $\mathrm{Cl}=$ confidence interval.

* Weighted percentages might not sum to $100 \%$ because of rounding.

$\dagger$ Includes regular soda, fruit drinks, sweet tea, and sports or energy drinks.

$\S$ Values for all states were different by chi-square test $(p<0.05)$.

" Missing data: $0.4 \%$ for employment status and $0.2 \%$ for education.

environments, including access and availability, cultural norms $(7,8)$, and advertising. This study found higher prevalences of daily SSB intake among younger adults, men, blacks, and persons with lower levels of education, which is consistent with previous reports that used data from the 2009-2010 NHANES (3) and 2011 and 2012 BRFSS (6,9). Adults with less knowledge about the adverse health consequences of SSB intake (5) might in part account for the higher reported consumption in some populations. Daily SSB intake was higher among young adults and unemployed adults but lower among older adults and retired adults in this analysis; previous studies based on NHANES and BRFSS data have found that older adults have lower SSB intake $(3,6,9)$.

The findings in this report are subject to at least three limitations. First, because BRFSS data are self-reported, they are subject to recall and social desirability bias, which might have underestimated or overestimated SSB intake. Second, only half of states elected to use the module; thus the

\footnotetext{
http://www.aacorn.org/RepoRese-2542.html.
}

findings might not be generalizable to the entire U.S. adult population. Finally, SSB intake was measured in frequency rather than volume of consumption; therefore, estimating the amount of SSBs consumed or the caloric intake from SSBs was not possible.

The frequency of daily SSB intake is high among adults, especially among certain subpopulations, as well as persons living in southern states. SSBs can contribute to obesity, type 2 diabetes, and cardiovascular disease related to excess intake of added sugars and calories from SSBs (2). SSB intake has been positively associated with markers of inflammation and insulin resistance, which might increase risk for cardiovascular disease and diabetes independently of obesity (2). Considering potential adverse health effects of SSB intake and the substantial contribution that SSBs make to excess dietary sugar, continuation of public health efforts aimed at decreasing high SSB intake is important. Actions can include education and awareness initiatives, increasing access to and promotion of healthier options through nutrition standards, 


\section{Summary}

What is already known about this topic?

Sugar-sweetened beverages (SSBs) are significant sources of added sugars among U.S. adults. SSB intake differs by geographical region and sociodemographic characteristics.

What is added by this report?

Data from the SSB intake module in the 2013 Behavioral Risk Factor Surveillance System provide the most recent state-specific data on consumption of SSBs. Among the 23 states surveyed and the District of Columbia, adult SSB intake was highest in Mississippi (47.5\%), followed by Louisiana (45.5\%) and West Virginia (45.2\%). At least once daily SSB intake was most common among persons aged $18-24$ years (43.3\%), men (34.1\%), non-Hispanic blacks (39.9\%), persons who reported not being employed (34.4\%), and persons with less than high school education (42.4\%).

What are the implications for public health practices? Because of the potential adverse health outcomes associated with SSB intake, including obesity, type 2 diabetes, and cardiovascular disease, public health practitioners should continue efforts aimed at decreasing SSB intake among demographic groups with the highest reported consumption. Strategies can include education initiatives, increasing access to healthier options through nutrition standards, increasing availability of drinking water in schools and public venues, screening and counseling patients on SSB reduction, and facility food and beverage changes in clinic or hospital settings for employees, families, and patients.

including food service guidelines, ${ }^{* *}$ and increasing the availability and promotion of drinking water in schools and public venues. ${ }^{\dagger \dagger}$ In addition, health care providers can screen and counsel patients on SSB reduction and support facility food and beverage changes in their clinic or hospital settings for employees, families, and patients. $\$ \$$

\footnotetext{
** http://www.cdc.gov/obesity/strategies/food-serv-guide.html.

${ }^{\dagger \dagger}$ https://www.planning.org/research/publichealth/pdf/wateraccessreport.pdf.

$\$ \$$ http://www.cdc.gov/obesity/strategies/healthy-hospital-environment-toolkit/ index.html.
}

\section{Acknowledgments}

BRFSS state coordinators; Suzianne Garner and Liping Pan, Division of Nutrition, Physical Activity, and Obesity, National Center for Chronic Disease Prevention and Health Promotion, CDC.

${ }^{1}$ Division of Nutrition, Physical Activity, and Obesity, National Center for Chronic Disease Prevention and Health Promotion (NCCDPHP), CDC; ${ }^{2}$ Division of Population Health, NCCDPHP, CDC.

Corresponding author: Sohyun Park, spark3@cdc.gov, 770-488-5163.

\section{References}

1. Drewnowski A, Rehm CD. Consumption of added sugars among US children and adults by food purchase location and food source. Am J Clin Nutr 2014;100:901-7. http://dx.doi.org/10.3945/ajcn.114.089458

2. Malik VS, Popkin BM, Bray GA, Després J-P, Hu FB. Sugar-sweetened beverages, obesity, type 2 diabetes mellitus, and cardiovascular disease risk. Circulation 2010;121:1356-64. http://dx.doi.org/10.1161/ CIRCULATIONAHA.109.876185

3. Kit BK, Fakhouri TH, Park S, Nielsen SJ, Ogden CL. Trends in sugarsweetened beverage consumption among youth and adults in the United States: 1999-2010. Am J Clin Nutr 2013;98:180-8. http://dx.doi. org/10.3945/ajcn.112.057943

4. Park S, McGuire LC, Galuska DA. Regional differences in sugar-sweetened beverage intake among US adults. J Acad Nutr Diet 2015;115:1996-2002. http://dx.doi.org/10.1016/j.jand.2015.06.010

5. Park S, Onufrak S, Sherry B, Blanck HM. The relationship between health-related knowledge and sugar-sweetened beverage intake among US adults. J Acad Nutr Diet 2014;114:1059-66. http://dx.doi.org/10.1016/j. jand.2013.11.003

6. Kumar GS, Pan L, Park S, Lee-Kwan SH, Onufrak S, Blanck HM. Sugar-sweetened beverage consumption among adults_-18 states, 2012. MMWR Morb Mortal Wkly Rep 2014;63:686-90.

7. Martin-Biggers J, Yorkin M, Aljallad C, et al. What foods are US supermarkets promoting? A content analysis of supermarket sales circulars. Appetite 2013;62:160-5. http://dx.doi.org/10.1016/j.appet.2012.12.001

8. McCabe-Sellers BJ, Bowman S, Stuff JE, Champagne CM, Simpson PM, Bogle ML. Assessment of the diet quality of US adults in the Lower Mississippi Delta. Am J Clin Nutr 2007;86:697-706.

9. Park S, Pan L, Sherry B, Blanck HM. Consumption of sugar-sweetened beverages among US adults in 6 states: Behavioral Risk Factor Surveillance System, 2011. Prev Chronic Dis 2014;11:E65. http://dx.doi.org/10.5888/ pcd11.130304 